OAI-PMH: http://www.indteca.com/ojs/index.php/Revista Scientific/oai

Artículo Original / Original Article

\title{
Éxodo del docente universitario en Venezuela
}

Autoras: Gainioska Dairesky Linarez Veloz Universidad Pedagógica Experimental Libertador, UPEL gainiosk1024@hotmail.com Caracas, Venezuela https://orcid.org/0000-0002-6307-001X

Gaidibeth Dubraska Linarez Veloz Universidad Pedagógica Experimental Libertador, UPEL gaidibethlinarez@gmail.com

Caracas, Venezuela https://orcid.org/0000-0002-7229-8630

\section{Resumen}

Esta investigación presenta una reflexión relacionada con el éxodo del docente universitario en Venezuela, que ha provocado la migración masiva del profesor universitario, generando preocupación y una crisis que responde al personal de profesores, estudiantes, empleados y trabajadores, quienes se dedican a desarrollar actividades académicas, investigación y extensión, es decir, en el campo científico, cultural y técnico. De esta manera se mantiene la autonomía, libertad de las universidades nacionales y experimentales. Para expresar los problemas causados en el docente universitario, se utiliza una metodología teórica, a través de un enfoque de estudio descriptivo-explicativo para la recopilación de información. La técnica es de tipo documental tomada de los medios y las opiniones de los docentes para la búsqueda de un análisis de la función y situación del profesorado. Los resultados reflejan dicho análisis, las relaciones a nivel conceptual, el aspecto social y económico y sus incidencias en la vida personal de los actores.

Palabras clave: éxodo intelectual; docente; universitario.

\section{Cómo citar este artículo:}

Linarez, G., \& Linarez, G. (2019). Éxodo del docente universitario en Venezuela. Revista Scientific, 4(14), 141-162, e-ISSN: 2542-2987. Recuperado de: https://doi.org/10.29394/Scientific.issn.25422987.2019.4.14.7.141-162

Fecha de Recepción: 17-05-2019
Fecha de Aceptación: 10-08-2019
Fecha de Publicación: 05-11-2019 


\title{
Exodus of the university professor in Venezuela
}

\begin{abstract}
This research presents a reflection related to the exodus of the university professor in Venezuela, which has caused the massive migration of the university professor, generating concern and a crisis that responds to the staff of professors, students, employees and workers, who are dedicated to developing academic activities, research and extension, that is, in the scientific, cultural and technical field. In this way the autonomy, freedom of national and experimental universities is maintained. To express the problems caused in the university teacher, a theoretical methodology is used, through a descriptiveexplanatory study approach for the collection of information. The technique is of documentary type taken from the media and the opinions of the teachers for the search of an analysis of the function and situation of the teaching staff. The results reflect this analysis, the relationships at the conceptual level, the social and economic aspect and their impact on the personal life of the actors.
\end{abstract}

Keywords: brain drain; teacher; university.

How to cite this article:

Linarez, G., \& Linarez, G. (2019). Exodus of the university professor in Venezuela. Revista Scientific, 4(14), 141-162, e-ISSN: 2542-2987. Recovered from: https://doi.org/10.29394/Scientific.issn.2542$\underline{2987.2019 .4 .14 .7 .141-162}$

Date Received:

17-05-2019
Date Acceptance:

10-08-2019
Date Publication:

05-11-2019 


\section{Introducción}

La temática que se aborda apunta a analizar, el éxodo del docente universitario en Venezuela, donde el desarrollo de la academia es el resultado de la relación social conocida como "educación", y se refiere a crecimiento personal, obtención de título, empleo, liderazgo, desarrollo intelectual, profesión, cambio de status, entre otros; persiguiendo como fin optimo la felicidad suprema del ser humano, satisfacción integral de sus necesidades.

En relación con los sueldos de los docentes, en estudios realizados por la Federación Venezolana de Maestros (FVM, 2015a), publicado en el periódico El Impulso, se consiguió que: Venezuela: 41,77\$ (SIMADI, Valor referencial 170Bs/\$), SICAD unificado $12.30 \mathrm{Bs} / \$: 569,10 \$$ (Este sistema no ha arrancado su oferta oficial), CENCOEX: 1.111\$ (Especialistas sostienen que esta tasa de 6,30 Bs/\$ no es una referencia real en el país) (pág. 1).

Todas estas referencias con respecto a Venezuela fueron hasta el 17 de febrero del año 2015, ya que el Presidente de la República, estableció una nueva tasa de cambio que afecta aún más el sueldo de un docente universitario en el país. Más aún cuanto, según la misma FVM antes citada, refiere que existe gran desproporción en comparación con los sueldos de otros países tales como: Colombia $600 \$$, Chile: $700 \$$, México: $1.340 \$$, Argentina: $1.375 \$$, entre otros.

Como se puede observar estos parámetros no están acordes con la realidad del docente venezolano, si no que por el contrario se ha conseguido en la investigación que existen grandes deficiencias, carencias, tanto en el aspecto económico como social, donde se evidencia que la relación educación y el desarrollo no se compagina con la situación actual, donde los sueldos están por debajo de los países más pobres como Haití y República Dominicana, ya que los docentes universitarios devengan un sueldo que está por debajo de los seis dólares (6\$). Dicha situación no solo afecta a las universidades, sino también es un estado de crisis general y global que 
produce un alto grado de pobreza en la población.

En este mismo orden de ideas, La Organización de las Naciones Unidas para la Educación, la Ciencia y la Cultura (UNESCO, 1998), plantea en el art. 16, de la Fuga de Cerebros a su retorno que:

Sería preciso poner freno a la "fuga de cerebros" ya que sigue privando a los países en desarrollo y a los países en transición, de profesionales de alto nivel necesarios para acelerar su progreso socioeconómico. Habría que atender a la creación de un entorno que atraiga y retenga el capital humano cualificado, mediante políticas nacionales o acuerdos internacionales que faciliten el retorno, permanente o temporal, de especialistas e investigadores muy competentes a sus países de origen (pág. $3)$.

En relación a lo planteado, se puede considerar que a pesar de este planteamiento de la UNESCO, las investigaciones científicas, que se realizan en las universidades con los estudiantes y publicadas mediante la extensión académica, han disminuido notablemente por reducción del presupuesto universitario para infraestructura, equipos y nuevas investigaciones. Aun existiendo todas estas dificultades, las universidades actualmente siguen siendo las que producen mayor cantidad de investigación científica en Venezuela.

Por consiguiente, el cerco económico que existe en las Universidades en Venezuela, obstaculiza su crecimiento, reduce la investigación y extensión, e incrementa la inequidad social, pues sin recursos para becas y ayuda estudiantiles adecuadas, los estudiantes que vienen de los sectores de mayor pobreza no pueden concluir la educación universitaria; y además con insuficiente dotación, no se podrán crear nuevas carreras ni habrá suficiente personal calificado, lo cual imposibilita el aumento de la oferta académica de cupos para cubrir la demanda con una educación de calidad.

Por otro lado, los docentes, manifiestan su malestar por la ausencia de políticas públicas orientadas a mejorar la situación económica y social de este 
profesional lo cual los hace sentirse en condiciones indignas por cuanto sus salarios están muy por debajo del sueldo mínimo lo cual no les permite ni siquiera poder obtener la canasta básica para su subsistencia, situación que ha provocado el éxodo o migración de valiosos profesionales a otros países con el fin de garantizar la satisfacción de sus necesidades básicas y académicas.

El éxodo de los docentes universitarios, objeto de este estudio no escapa a los cambios e innovaciones que actualmente presenta el país, para lo cual se hace necesaria una educación cada vez más dinámica, donde se concrete la participación activa de todos los educandos, especialmente de los docentes de esta institución, quienes presentan las mismas necesidades y exigencias, lo cual se detecta a través de la información recogida por la autora en entrevistas informales aplicadas a los docentes de esta casa de estudio.

De aquí se puede inferir que las universidades en Venezuela deben estar comprometidas con la actualización, capacitación y perfeccionamiento del docente, tal como lo indica en su visión y misión.

Derivado de lo anteriormente expresado surgen las interrogantes de la presente investigación: ¿Cuál es la situación social del docente universitario en Venezuela?; ¿Cuál es la situación económica del docente universitario en Venezuela?; ¿Existen motivos para el éxodo del docente universitario en Venezuela?; ¿Existen actualmente estrategias de capacitación y perfeccionamiento que estimulen el crecimiento intelectual del docente universitario en Venezuela?

\section{Metodología}

En esta investigación se utilizó una metodología teórica, a través de un enfoque de estudio descriptivo-explicativo para la recopilación de información. La técnica es de tipo documental tomada de los medios y las opiniones de los docentes para la búsqueda de un análisis de la función y situación de los 
mismos, y en ella se plantea la problemática generada al profesor universitario, debido al éxodo en Venezuela. La recolección de información se obtiene de manera documental proveniente de los medios de comunicación, donde los contenidos de éstos, permitieron hacer un análisis de la función y situación del profesorado, los cuales afrontan circunstancias negativas en cuanto a su situación social y económica.

Al respecto, Sandín (2003), citado por Butrón y Calderón (2012), considera al método fenomenológico-hermenéutico, como: "las descripciones detalladas de situaciones, eventos, personas, interacciones y comportamientos observados, incorporando la voz de los participantes, sus experiencias, actitudes, creencias, pensamientos y reflexiones, tal como son expresadas por ellos mismos" (pág. 87).

Como se puede observar, esta investigación se fundamentó en el acontecer del profesor universitario relacionado con su comportamiento migratorio tomando en consideración el aspecto social y económico y la situación que prevalece en Venezuela.

En este mismo orden de ideas, se puede indicar que el análisis permitió realizar un intercambio en el desarrollo de la fenomenología educativa, proyectando las impresiones, experiencias y saberes que dan respuesta a las interrogantes que se formularon en relación al éxodo del docente universitario en Venezuela; motivo por el cual, la investigación cualitativa, ayudó a comprender las acciones de vida, la cultura, y el desenvolvimiento, de estos profesionales al interactuar permanente con lo demás.

\section{Contexto}

El área de estudio se ubicó en los docentes del sector universitario en Venezuela. Fundamentalmente se analizó el sector de los actores relacionados con el éxodo del docente universitario. En relación a esta propuesta de selección de informantes, Martínez (2004), citado por Grimaldo 
(2009), quien plantea algunos criterios para la realización de una investigación cualitativa, tales como:

...la información hay que buscarla donde está... la observación no debe deformar, distorsionar o perturbar la verdadera realidad del fenómeno que se estudia. Tampoco debe descontextualizar los datos aislándolos de su entorno natural. Todo esto exige que la información sea recogida en la forma más completa posible... (pág. 4).

Al respecto para este estudio, las fuentes de información estuvieron constituidas por textos especializados, la participación social, reportes de investigación, publicaciones en la red, entre otros, obteniendo mediante esta esta vía conocimiento sistemático, de la realidad estudiada.

Además, para apoyar el trabajo de campo, se usaron los guiones de entrevista. De igual manera la información recabada se reprodujo fielmente por las investigadoras para darle significado mediante el método hermenéutico elegido para tal fin.

\section{Análisis de Información}

El análisis se realizó a través de la revisión bibliográfica exhaustiva, entrevistas, entre otros; todo esto relacionado con la fuga del talento humano docente universitario en Venezuela. Posteriormente, ejecutaron la integración de categorías emergentes, producto de la identificación de los elementos, frases o fragmentos importantes de los datos aportados por los actores, los cuales fueron agrupados, conceptualizados y codificados de manera irrefutable a fin de constituir un grupo notorio fabricado por los actores sociales relacionados con el tema investigado.

En lo que respecta a sus beneficios, tanto económicos como sociales, se produce un efecto negativo en la educación universitaria, debido a que toda esta problemática genera desmotivación al momento de seguir ejerciendo su carrera como profesionales, al no tener el derecho de disfrutar de sus 
beneficios, por ser mal pagados y más aún que son los menos tomados en cuenta en lo que respecta a sus necesidades.

Así mismo, con todo este tipo de adversidades que se presentan dentro de este ámbito, sino se toman cartas en el asunto para mejorar esta crisis con el paso del tiempo los profesores podrían tomar la decisión de no asistir más a sus puestos laborales porque ellos también son humanos y profesionales, y merecen así como muchos un ritmo de vida justa, donde puedan tener al menos un seguro y un sueldo digno con el que puedan subsistir en la actualidad, ya que se han evidenciado factores que inciden directamente en ellos: bajos salarios, no dignos para el trabajo que realizan, problemas con su seguro social, seguro de hospitalización, cirugía y maternidad (HCM) y contratación colectiva, entre otros.

Todo lo mencionado anteriormente trae como consecuencia el descontento de los educadores universitarios, ocasionando abandono en los puestos de trabajos ya que no se sienten cómodos con los beneficios que se les brinda, y sin su presencia las nuevas generaciones no podrían estar en la capacidad suficiente para desarrollarse, al no recibir la preparación adecuada, ocasionando así un deterioro no solo en la educación si no en la sociedad y ética profesional.

\section{Resultados alcanzados}

Ante esta situación, en Venezuela, la profesión docente, degradada y mal remunerada, presenta grandes déficits en relación con las necesidades, la falta de reposición oportuna de los cargos y los pésimos salarios, lo que representa la causa de la escasez de docentes calificados. A partir de esta problemática Rachadell (2013): en carta enviada al Prof. Víctor Márquez, Presidente de la Asociación de Profesores de la Universidad Central de Venezuela (APUCV), representante legal y legítimo del profesorado universitario de Venezuela, tratando de reafirmar la validez y vigencia de las 
Normas de Homologación (NH); donde se planteó ante el Ministerio de Educación Universitario un petitorio de reivindicaciones ajustadas a dichas $\mathrm{NH}$, las cuales han sido violentas desde el año 2004 (págs. 2-19).

A pesar de que los gremios, representantes de los docentes siguieron ejerciendo acciones, ante las diferentes autoridades del Ministerio de Educación Superior, nunca se ajustó el salario desde el año 2004 hasta el año 2014, no se nombró la comisión del Consejo Nacional de Universidades (CNUFAPUV), y el salario de los profesores instructores a tiempo completo (primer escalafón de la carrera académica) descendió a nivel del salario mínimo. En el año 2013 se discutió y aprobó la Primera Convención Colectiva del Sector Universitario (ICCU), sin la participación del gremio de profesores universitarios. Se impone un ajuste salarial insuficiente y los derechos del personal jubilado, pensionado y sobreviviente fueron gravemente lesionados, al introducirse el concepto de pensión indivisible para rebajar las pensiones en comparación con el salario del personal en servicio.

Estas violaciones recurrentes a la libertad sindical, a derechos adquiridos y al derecho constitucional de las condiciones de remuneración y trabajo de los profesores universitarios, fue la causa del paro nacional del año 2013, de conde surgió un acuerdo suscrito entre La Federación de Asociaciones de Profesores Universitarios de Venezuela (FAPUV), la Asociación Venezolana de Rectores Universitarios (AVERU) y el Ministerio del Poder Popular para la Educación Universitaria, Ciencia y Tecnología (MPPEUCT), donde se reconoció a la Federación como legítima representante de los profesores universitarios; corroborando el avance e inaccesibilidad de los derechos laborales de los profesores universitarios, reconociendo la vigencia de los acuerdos federativos, Normas de Homologación y Actas Convenios Institucionales, suscritas entre las Universidades y sus Asociaciones de Profesores.

Refiere el mismo autor que a pesar de los logros anteriores, en los años 
2015-2016, el salario de los dos primeros escalafones de la carrera académica, Instructor, Asistente y de los Auxiliares Docentes, quedó por debajo del salario mínimo. Luego, la FAPUV participó en la discusión de la II Convención Colectiva del Consejo Universitario (IICCU), planteando ésta, que se debían incorporar en la discusión a las autoridades universitarias, en representación de las Universidades Autónomas, y se debían respetar los acuerdos preexistentes y las Normas de Homologación.

De igual manera en la referida convención, hubo un ajuste salarial para el sector universitario por decreto presidencial; quedando la tabla del personal académico con diferencias de apenas 1 y $2 \%$ entre los tres primeros niveles del escalafón (Instructor, Asistente y Agregado), dicho ajuste no cubre la canasta alimentaria en ninguno de los cargos, ni garantiza el cumplimiento de la jornada de trabajo en las instituciones.

El criterio para establecer dicho ajuste no fue la inflación acumulada, como establecen las $\mathrm{NH}$, no fue tampoco el costo de la canasta básica, como se establece en el Título III: de los Deberes, Derechos Humanos y Garantías, capítulo V, de los Derechos Sociales y de las Familias de la Constitución de la República Bolivariana de Venezuela (1999), la cual reza lo siguiente:

El Estado garantizará a los trabajadores y trabajadoras del sector público y del sector privado un salario mínimo vital que será ajustado cada año, tomando como una de las referencias el costo de la canasta básica. La Ley establecerá la forma y el procedimiento (art. 91).

De igual manera, no hay transparencia en el manejo de los recursos, que no son del gobierno, son de la educación; además las prestaciones sociales por el tiempo de servicio prestado no se cancelan a tiempo, lo cual ocasiona una gran deuda en las Universidades Nacionales. A unos se les deben todas las prestaciones, a otros se les ha pagado la antigüedad y se les 
deben los intereses y en cuanto a los fideicomisos que manda la ley no se han creado y al término de la relación laboral hay que esperar muchos años para cobrar.

También, los Institutos de Previsión Social del Profesorado de las Universidades Nacionales, creados para garantizar la seguridad social de los profesores universitarios, presentan déficits crecientes que están debilitando la red de protección que ellos ofrecen, debido a la reducción sistemática de los recursos que deben financiar tan importante derecho y al deterioro del salario real del profesorado que contribuye con su funcionamiento.

Se ha discriminado a los profesores universitarios en cuanto a las asignaciones para HCM, y otros servicios, en relación al resto del personal universitario (administrativos, obreros), usurpando contribución del patrono a los Fondos de Pensiones y Jubilaciones. Además, en algunas universidades no se les permite a los profesores la afiliación al Instituto Venezolano de los Seguros Sociales (IVSS) y la pensión de vejez, porque no fueron inscritos oportunamente por las Universidades y porque no se contempla este concepto en el presupuesto universitario.

Ante esta situación los profesores universitarios en Venezuela confrontan una crisis tremenda en cuanto al éxodo de docentes calificados, ya que esta coyuntura país no satisface sus necesidades básicas y de acuerdo a Márquez (2019), presidente de la Asociación de Profesores de la Universidad Central de Venezuela (APUCV), quien señaló que: "aproximadamente $50 \%$ de la población docente de todas las universidades han salido del país, producto de la crisis económica y política" (pág. 1).

Todo esto es debido a que los recursos de las universidades no son suficientes para avalar su crecimiento personal y profesional, de acuerdo a sus aspiraciones individuales en cuanto a seguridad, dificultades socioeconómicas, tales como: aumento del costo de la vida, desocupación, entre otros, lo cual se traduce en desesperanza e incertidumbre, obligándolos a ir al 
encuentro de opciones superiores en relación a calidad de vida en el exterior.

Todas las razones mencionadas han ocasionado el éxodo masivo del profesor(a) universitario, generando preocupación, y afectando a su vez a una gran comunidad de profesores, estudiantes, empleados y obreros. Dichas dificultades generadas por la problemática-país actual ha sido explosiva en el grupo familiar en el aspecto socio-económico y político, lo cual ha obligado a los docentes a emigrar debido al estado de indefección al que han sido sometidos por parte del gobierno, quien ni remotamente tiene la disposición para solventar estas circunstancias, motivo por el cual a los profesionales jóvenes al graduarse no les queda otra opción que irse del país a buscar otros horizontes seguros con calidad de vida y en general un futuro más promisorio.

Frente a esta perspectiva, el éxodo del docente universitario significa el desafío mayor que tienen las universidades venezolanas actualmente, ya que esto indica la negación del desarrollo científico-tecnológico mundial ocasionando un deterioro al pueblo venezolano irrecuperable en el tiempo.

Al respecto, Ramírez (2015), Coordinador del doctorado en Educación de la Universidad Central de Venezuela (UCV), citado en la Federación Venezolana de Maestros (FVM, 2015b), señala que: en la UCV y la Universidad Simón Bolívar (USB): "se han registrado aproximadamente 600 renuncias" (pág. 1); desde el año 2012, lo cual se debe a que la profesión docente no ha sido valorada en Venezuela en cuanto a ingresos, siendo ésta la principal causa del déficit económico y las pocas opciones de empleo, lo cual provoca la diáspora en las universidades. También afirma Ramírez (2015), citado por la FVM (2015c), que existen otras razones que ocasionan el éxodo del docente universitario; tales como: "no hay posibilidades en el país de intercambio científico a nivel internacional ni la posibilidad de estudios de perfeccionamiento, las universidades han cerrado sus programas de financiamiento, congresos y postgrados, lo cual repercute en una persona proactiva y capacitada" (pág. 1). 
Todo esto se refleja en la situación social y económica compleja que confronta el docente universitario al permanecer inmerso en dicha problemática, por cuanto esto repercute en la calidad educativa y seguridad social de dicho profesional, razón por la cual, Pérez (2018), citado por López (2019), en el artículo titulado La Pedagogía del Amor y la Ternura: Una Práctica Humana del Docente de Educación Primaria, donde refiere:

La reconstrucción de Venezuela va a necesitar de educadores corajudos, valientes, creativos, que asuman la educación como un medio fundamental para producir vida abundante para todos. Estamos en la sociedad del conocimiento y hay un consenso generalizado a nivel mundial de que la educación es el medio fundamental para combatir la violencia, construir ciudadanía y lograr un desarrollo humano sustentable (pág. 271).

Aquí se puede inferir, que el docente universitario al emigrar, busca resolver el conflicto en cuanto a calidad de vida, encarecimiento de medicinas, salud, desocupación, riesgo de inseguridad que confronta en el país. Por este motivo, la Asociación de Profesores de la Universidad Pedagógica Experimental Libertador (UPEL), ante el atropello continuo a los derechos de los docentes universitarios, que actualmente se ha agravado y al no cancelarse puntualmente su salario, porque el ejecutivo nacional no envía regularmente los recursos a la universidad (presupuesto deficiente), dicho gremio ha manifestado un profundo rechazo a esta situación, presentando una serie de acciones adecuadas a fin de defender los derechos de sus agremiados, generando soluciones que impidan el colapso de dicha institución libre, democrática, autónoma y de calidad.

\subsection{Análisis de resultados}

El siguiente análisis, se refiere a la fase o etapa de desarrollo del diagnóstico de la situación encontrada durante la realización de la 
investigación, la misma se genera una vez aplicado el instrumento de recolección de información, el cual tuvo como entre sus principales propósitos determinar el éxodo del docente universitario en Venezuela, para lo cual se utilizó el método estadístico de análisis, por lo que se estableció una frecuencia absoluta y porcentual en la medida que se obtuvieron las respuestas.

Estas opiniones dadas por el personal docente son un indicador del panorama encontrado, después de organizarlas, se procesaron a través del software estadístico SPSS, versión 15, o en su defecto en una hoja de cálculo de Microsoft Excel, después fueron vaciados en cuadros estadísticos y graficas de barras para un mejor manejo visual donde se muestra el estudio de cada indicador. La interpretación de los resultados se hizo de forma descriptiva agrupando algunos ítems relacionados, haciendo énfasis en las alternativas de más altos porcentajes.

En relación a la cantidad de sujetos se aplicó a veinte (20) docentes de la UPEL, quienes son los afectados de la situación problema estudiada en la presente investigación, destacando los siguientes resultados:

Cuadro 1. Valores en frecuencia y porcentaje de los resultados obtenidos en la dimensión Social, indicador: Seguridad Laboral.

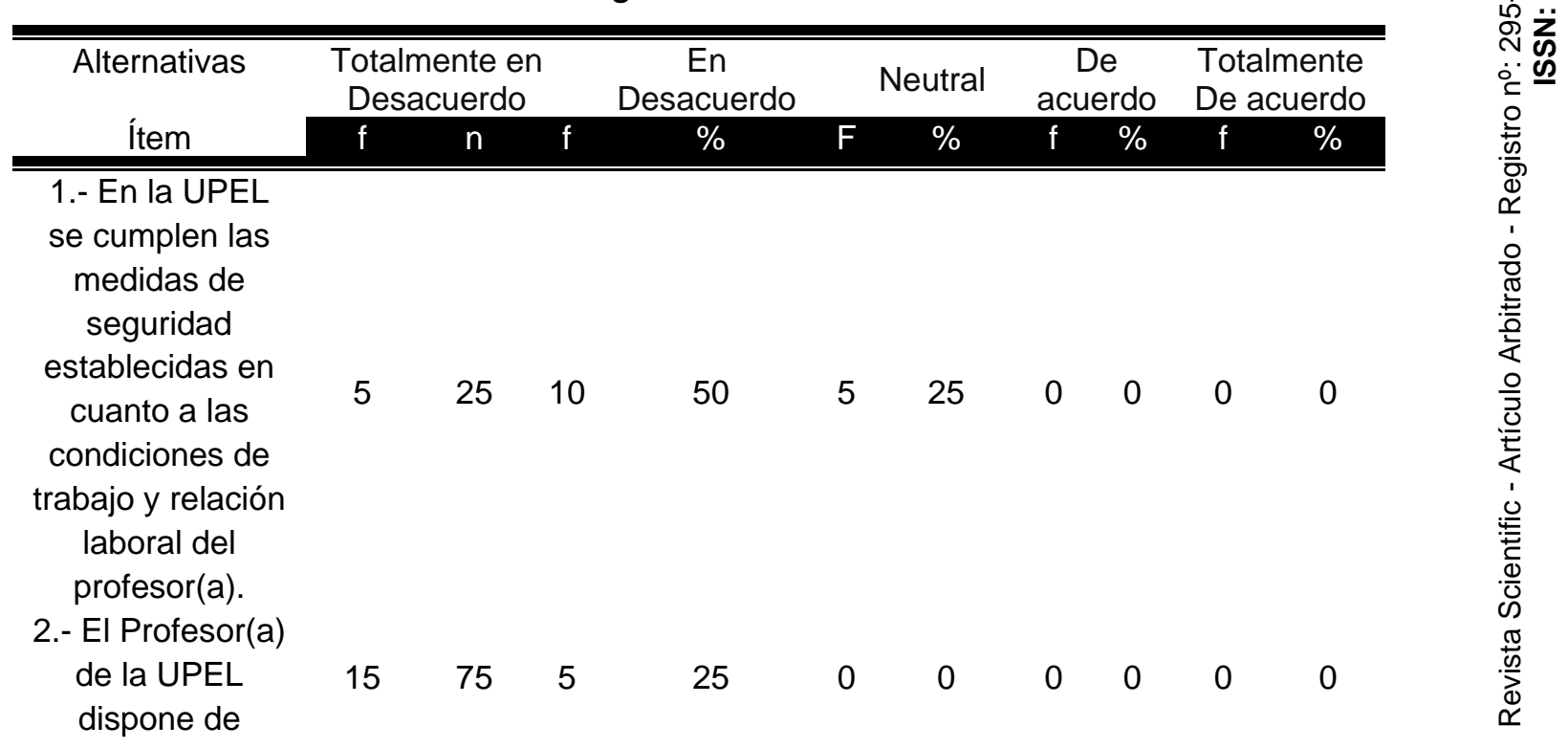


recursos para la

seguridad laboral

ajustados a la

realidad actual.

\begin{tabular}{llllll}
\hline Promedio & $50 \%$ & $40 \%$ & $10 \%$ & 0 & 0 \\
\hline \hline
\end{tabular}

Fuente: Las Autoras (2019).

Gráfico 1. Representación del indicador Seguridad Laboral.

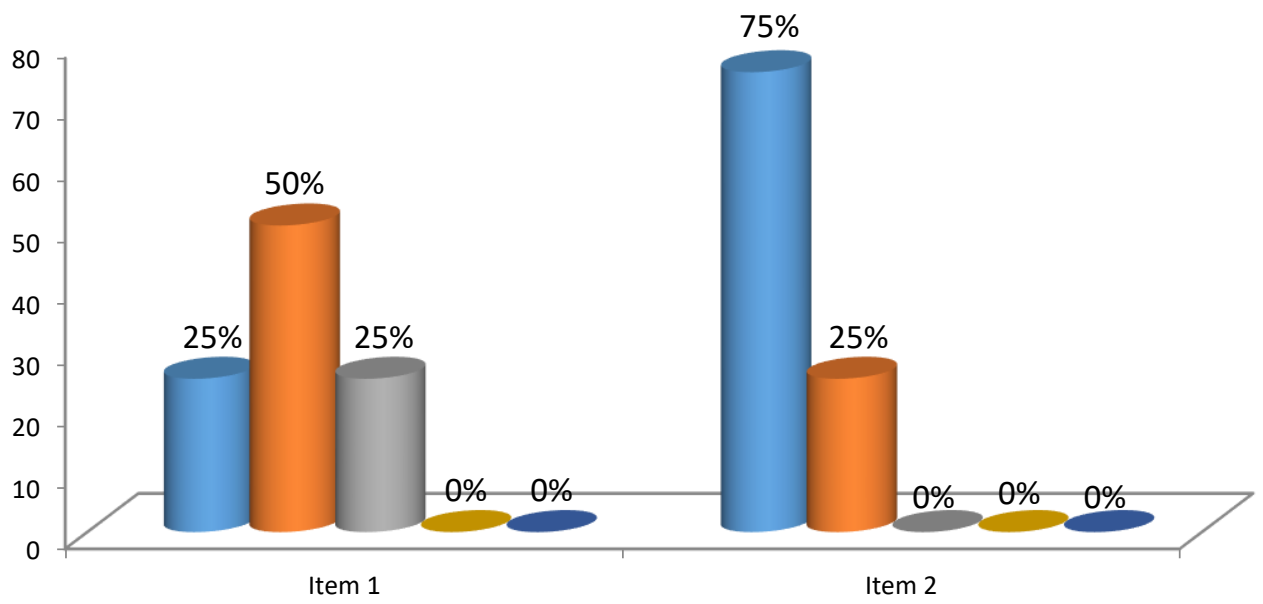

Fuente: Las Autoras (2019).

Los datos obtenidos mediante el estudio diagnóstico, referidas a la dimensión Social, indicador: Seguridad Laboral, arrojaron lo siguiente:

En el cuadro 1 y gráfico 1, referido a la información suministrada por los docentes, en el ítem 1, en cuanto al hecho de si en la UPEL se cumplen las medidas de seguridad establecidas en para las condiciones de trabajo y relación laboral del profesor(a), el 50\% de los sujetos encuestados, señalaron estar en desacuerdo con esta proposición, el $25 \%$ estuvo totalmente en desacuerdo y el otro $25 \%$ neutral. Estos resultados evidencian la sentida necesidad por todos los sujetos investigados de que se cumplan cabalmente las condiciones laborales de los mismos en dicha casa de estudio, ya que de 
ellas dependen en gran parte el éxito de la praxis docente.

Por su parte el ítem 2, donde se indaga si el Profesor(a) de la UPEL dispone de recursos para la seguridad laboral ajustados a la realidad actual, el $75 \%$ de los docentes manifestaron estar totalmente en desacuerdo y el $25 \%$ en desacuerdo. En general estas respuestas en promedio se inclinan hacia la tendencia negativa dejando claro que no disponen de salarios ajustados a la realidad que vive Venezuela en todos los ámbitos, lo que genera que el rol del educador se vea perturbado por dicha situación.

Dichos resultados, permitieron deducir que no se están cumpliendo las medidas de seguridad establecidas en relación a las condiciones laborales de los docentes, no contando la institución con los recursos necesarios para garantizar dicha seguridad, lo cual es una debilidad para la institución.

Aquí se observa, que la profesión docente es una de las menos valoradas por el estado venezolano, en lo que a salario se refiere; lo cual constituye el duro escollo de la crisis económica y las reducidas oportunidades de desarrollar el ejercicio docente; posibles causas que han generado el fenómeno del éxodo, en búsqueda de seguridad laboral, motivo por el cual surge la necesidad de que los docentes cuenten con programas que conlleven a brindar respuestas a la realidad de su situación y la educación en sentido general, ya que es importante mantener incentivos justos por la labor que ejercen estos profesionales, recuperando así, gran parte las políticas públicas que no se cumplen actualmente, por falta de recursos presupuestarios.

\section{Conclusiones}

Se determinó la situación en relación al éxodo de los docentes universitarios los cuales actualmente están atravesando una profunda crisis en todos los ámbitos, producto de la situación que enfrenta Venezuela, requiriendo entonces de nuevas políticas que vayan a la par con la realidad. Asimismo se pudo conocer que no se están cumpliendo las medidas de 
seguridad establecidas en relación a las condiciones laborales, igualmente se evidencio que los profesores(as) no disponen de recursos para la movilidad social, ni horarios para asistir a encuentros científicos internacionales, mientras que en cuanto a la previsión social se presenta una debilidad porque el personal docente no tiene garantizado un seguro de hospitalización, cirugía y maternidad que este ajustado a la situación real, quedando desprotegido ante una emergencia de salud; lo cual representa la tendencia menos favorable para mejorar la actuación social de educador universitario.

De igual manera el estudio reveló principalmente el hecho de que los salarios devengados por los docentes no han logrado alcanzar los estándares mínimos como para poder compensar los niveles de inflación en el país. Sin embargo, se pudo observar que existen suficientes factores motivacionales que se traducen en un fuerte compromiso del maestro con su profesión, a pesar de sus elevados niveles de insatisfacción con los salarios y beneficios percibidos.

Por consiguiente, a pesar de que las universidades están comprometidas desde el punto de vista reglamentario con la actualización, capacitación y perfeccionamiento del docente universitario, con una visión prospectiva que contribuya de manera sistemática y eficiente con la formación de formadores, entes transformadores, autores y actores en la búsqueda de la formación de un profesional más eficiente, toda esta reglamentación no es suficiente, pues no se orienta con criterios y lineamientos precisos, relacionados con el cómo se va a realizar esa actualización y perfeccionamiento, que permitiría al docente universitario avanzar en el desarrollo de su carrera académica, ya que no cuenta con los suficientes recursos presupuestarios para cumplir con dicha reglamentación.

Por la razón antes mencionada, no existe un programa de desarrollo profesional que propicie en los docentes universitarios la necesidad de construir un plan de formación, para poder crear y recrear el conocimiento 
científico, pedagógico y social, basado en la investigación.

De igual manera, las universidades presentan limitaciones presupuestarias, y más aún ahora cuando el país presenta un déficit económico exponencial y del cual éstas no pueden escapar. Además, carece de una política sistemática y estratégica en materia de recursos humanos, situación que se observa en la apertura de concursos de oposición y cambios de dedicación que no satisfacen las necesidades reales de las instituciones mencionadas.

Ante esta situación se sugiere la realización de estudios especializados e instrucción permanente del docente universitario, lo cual se considera indispensable para alcanzar la excelencia en la educación y la ética científica, por cuanto las universidades requieren de un profesional altamente calificado para que pueda interactuar con su entorno a fin de ser aprovechado por la institución a la cual este adscrito.

A partir de esta premisa, se propusieron estrategias de capacitación y perfeccionamiento dirigidas al crecimiento intelectual de estos profesionales, a través de un programa que tiene como objetivo principal, servir como base para que los docentes puedan modificar los nuevos paradigmas de vida generados por la situación política y social que caracteriza a Venezuela, además de buscar herramientas que sirvan para mejorar el sistema educativo y así dar respuestas a diversos problemas que atraviesan las universidades del país.

Esta investigación es relevante por el hecho de que los resultados obtenidos servirán de ayuda para facilitar la comprensión de lo que significa el éxodo del docente universitario en Venezuela, en cuanto a la excelencia de dichas organizaciones, lo cual incidirá en beneficio de la sociedad, formando profesionales más calificados para los cambios y transformaciones que permitan al mismo tiempo reforzar el desarrollo humano y cultural del país. Por otro lado, este estudio presenta una serie de alternativas que posiblemente 
redundarán en beneficio de la situación crítica que confronta el docente universitario en Venezuela en cuanto al éxodo.

\section{Referencias}

Butrón, M., \& Calderón, L. (2012). El proceder fenomenológico y hermenéutico en la Construcción de Significados. Reflexionando una experiencia. Revista electrónica REDINE - UCLA, 2(7), 84-100, eISSN: 2244-7997. Recuperado de:

http://webcache.googleusercontent.com/search?q=cache:http://www.u cla.edu.ve/viacadem/redine/RevistaEREDINE/TrabajosTodos/PRevVol 2N72012Doc.doc

Constitución de la República Bolivariana de Venezuela (1999). Título III, de los Deberes, Derechos Humanos y Garantías. Capítulo V, de los Derechos Sociales y de las Familias. Gaceta Oficial Extraordinaria N ${ }^{\circ}$ 36.860 de fecha 30 de diciembre. Caracas, Venezuela: Asamblea Nacional Constituyente. Recuperado de:

https://www.oas.org/dil/esp/constitucion venezuela.pdf

FVM (2015a,b,c). Docentes emigran a otras latitudes: Fuga de cerebros afecta desarrollo académico. Barquisimeto, Venezuela: Nota de prensa de la Federación Venezolana de Maestros, publicada en el periódico El Impulso.com. Recuperado de:

https://www.elimpulso.com/2015/05/03/docentes-emigran-a-otraslatitudes-fuga-de-cerebros-afecta-desarrollo-academico/

Grimaldo, M. (2009). Manual de Investigación en Psicología. Capítulo: Investigación Cualitativa. Lima, Perú: Universidad Nacional Federico Villarreal. Recuperado de:

https://www.researchgate.net/publication/266260101 INVESTIGACIO N CUALITATIVA

López, C. (2019). La Pedagogía del Amor y la Ternura: Una Práctica 
Humana del Docente de Educación Primaria. Revista Scientific, 4(13), 261-277, e-ISSN: 2542-2987. Recuperado de:

https://doi.org/10.29394/Scientific.issn.2542-2987.2019.4.13.13.261$\underline{277}$

Martínez, M. (2004). Ciencia y Arte en la metodología cualitativa. México D.F.: Editorial Trillas.

Márquez, V. (2019). 50\% de profesores universitarios se ha ido del país por sus condiciones críticas. Caracas, Venezuela: Editorial Contrapunto, C.A. Recuperado de:

https://contrapunto.com/nacional/victor-marquez-50-de-profesoresuniversitarios-se-han-ido-del-pais-por-sus-condiciones-criticas/\#

Pérez, A. (2018). Educar en tiempos de crisis (I). Periódico del país, RIF: J30202528-1. [Documento en línea]. Zulia, Venezuela: Diario Panorama.

Recuperado de: https://www.panorama.com.ve/opinion/Educar-entiempos-de-crisis-I-por--Antonio-Perez-Esclarin-20180628-0100.html

Rachadell, M. (2013). Informe sobre la vigencia de las normas de homologación y sobre la inconstitucionalidad de las cláusulas $5^{a}$ y $6^{a}$ del proyecto de convención colectiva única para regular las relaciones laborales del personal universitario. Caracas, Venezuela: Asociación de Profesores de la Universidad Central de Venezuela APUCV. Recuperado de:

http://www.ciens.ucv.ve/ciens/wp-content/uploads/2013/06/Carta-aAPUCV.pdf

UNESCO (1998). Declaración Mundial sobre la Educación Superior en el siglo XXI: Visión y Acción. Conferencia Mundial sobre la Educación Superior en el siglo XXI: Visión y acción, Código del documento: ED.98/CONF.202/3. París, Francia: Organización de las Naciones Unidas para la Educación, la Ciencia y la Cultura. Recuperado de: http://www.unesco.org/education/educprog/wche/declaration spa.htm 
Gainioska Dairesky Linarez Veloz

e-mail: gainiosk1024@hotmail.com

en el año 2012 me gradúo de Magister Scientiarum en Educación Mención Desarrollo Comunitario en la Universidad Nacional Experimental de los Llanos Centrales Rómulo Gallegos (UNERG) de San Juan de Los Morros; en el año 2013 ingrese al programa de Doctorado en Ciencias de la educación en la UPEL-IPC, Caracas; Docente de aula en la Unidad Educativa Ayacucho, Barquisimeto (2004 al 2012); en el año 2012, ingrese a la UPEL-IMPM, Caracas, con el cargo de profesor ordinario a tiempo completo, en la Subdirección de Extensión; Coordinadora del Subprograma de Capacitación y Actualización del Personal Académico "Tutores"; en el año 2014, me traslado a la UPEL-IPB, Barquisimeto, con el Cargo de profesora a tiempo completo, en el departamento de Formación Docente; Reconocimientos y distinciones por destacado desempeño profesional; Curso de Inglés Instrumental para Estudios de Postgrado UPEL-IPB, Barquisimeto en el año 2017; Participación en eventos nacionales e internacionales: 2do Congreso Internacional e Interactivo de Educación, Herramientas Tecnológicas para el personal docente de Ciencias Sociales, entre otros. 


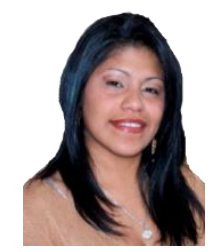

\section{Gaidibeth Dubraska Linarez Veloz e-mail: gaidibeth2014@gmail.com}

Nacida en Barquisimeto, estado Lara, Venezuela, el 22 de septiembre, 1973. Profesora Egresada de la Universidad Pedagógica Experimental Libertador (UPEL-IPB), Barquisimeto, en Educación Preescolar en el año 1999; Docente Il del Ministerio de Educación Superior (1999 hasta el 2006); Magister en Educación Superior, Mención Docencia Universitaria; Egresada de la Universidad Fermín Toro (UFT), Cabudare, estado Lara, en el año 2004; con Estudios Doctorales en la Universidad Pedagógica Experimental Libertador (UPEL-IPC), Caracas (2013 al 2018); Me Desempeñé en el cargo de docente ordinario en la Categoría de Agregado a Dedicación Exclusiva en la UPELIPB, Barquisimeto; Coordinadora de la Línea de Investigación en Competencias Investigativas en la UPEL-IMPM (2013 al 2017); Tutora Académica de Trabajos de Grado en Especialización en Educación Inicial de la UPEL-IMPM (2009 al 2016); Ponente Internacional en el II Congreso Internacional e Interactivo de Educación (CIEDUC), en el año 2013; Ponente Nacional en la II Jornada de Investigación de la Unidad de Investigación en Ciencias Funcionales "Dr. Haity Moussatché" (UNIHM) y la Universidad Centroccidental Lisandro Alvarado (UCLA), Barquisimeto, estado Lara, en el año 2012; Revisión de Expedientes en el año 2014; Jurado de los Concurso de Oposición Docente (UPEL-IMPM), en el año 2015; Curso de Inglés Instrumental para Estudios de Postgrado (UPEL-IPB), en el año 2017.

El contenido de este manuscrito se difunde bajo una Licencia de Creative Commons ReconocimientoNoComercial-Compartirlgual 4.0 Internacional 